\title{
Calidad de las guías de práctica clínica cubanas
}

\author{
Quality of the Cuban clinical practice guidelines
}

\author{
Ana Margarita Toledo Fernández,' Niviola Cabrera Cruz," Amaylid Arteaga \\ García,' Yoerquis Mejías Sánchez"'I \\ ' Doctor en Medicina. Máster en Atención Primaria de Salud. Especialista de II Grado \\ en Medicina General Integral. Viceministerio de Docencia e Investigaciones, MINSAP. \\ La Habana, Cuba. \\ "Doctor en Medicina. Especialista de II Grado en Epidemiología. Viceministerio de \\ Docencia e Investigaciones, MI NSAP. La Habana, Cuba. \\ "II Doctor en Medicina. Máster en Infectología. Especialista de I Grado en Pediatría. \\ Viceministerio de Docencia e Investigaciones, MINSAP. La Habana, Cuba.
}

\section{RESUMEN}

Se realizó una evaluación de la calidad de las guías de práctica clínica producidas en Cuba por las distintas especialidades, en formato impreso y en la red telemática de salud, con el propósito de identificar las debilidades que contienen que permitan confeccionar documentos metodológicos que contribuyan a su correcta elaboración. Se decidieron criterios de elegibilidad, de inclusión y de exclusión. Se revisaron 377 documentos. Se utilizó el Instrumento Internacional de Evaluación conocido como Appaisal of guidelines research and evaluation, el que fue aplicado por tres evaluadores para cada guía, si los resultados resultaran discordantes, se reevaluaba la guía por otros dos evaluadores independientes. Todos los evaluadores fueron previamente entrenados en el uso de instrumento. Algunas de las debilidades detectadas fueron: las guías no están realizadas por equipos multidisciplinarios, no se tuvo en cuenta el parecer de los pacientes, no dan respuestas a preguntas clínicas, no declaran objetivos, ni población diana ni criterios de inclusión ni de exclusión, entre otros. Se trabaja en la construcción de las Directrices Metodológicas para la Elaboración y Adaptación de Guías de Práctica Clínica en el Sistema Nacional de Salud.

Palabras clave: Guía de Práctica Clínica, calidad, evaluación. 


\title{
Revista Cubana de Salud Pública. 2011;37(3):349-358
}

\begin{abstract}
The quality of the Clinical Practice Guidelines made in Cuba by the various specialties was evaluated in print form and in the health telematic network, with the objective of identifying deficiencies in their drafting and thus drawing up methodological documents for their adequate preparation. Eligibility, inclusion and exclusion criteria were agreed upon. Three hundred seventy seven documents were reviewed. The International Evaluation Instrument known as Appraisal of guidelines for research and evaluation was used. Three evaluators were in charge of assessing each guideline; if results did not match, then the guideline was re-evaluated by another pair of outside evaluators. All these persons were previously trained in the use of the instrument. Some of the detected deficiencies were: guidelines were not prepared by multidisplinary teams, the criteria of patients were not taken into consideration, they did not respond to clinical questions and they did not state their objectives, target population or inclusion/exclusion criteria. We are working on the making up of the Methodological Instructions for the Preparation and Adaptation of the Clinical Practice Guidelines in the National Health Care System.
\end{abstract}

Key words: clinical practice guidelines, quality, evaluation.

\section{NTRODUCCI ÓN}

En 1948 la descripción de "ensayo clínico aleatorio", constituye el punto de partida para el desarrollo de una multitud de técnicas de estudio y análisis científicos basados en la estadística y la epidemiología clínica. Así es como, David Sackett, Archibald Cochrane, Iain Chalmers, R. Brian Inés, Gordon H. Guyatt T. Peter Tuqwell, todos médicos prácticos de avanzada, comprenden con claridad que al aplicar ciertas estrategias básicas de la epidemiología al estudio de sus pacientes, pueden mejorar sustancialmente su pericia en el diagnóstico, pronóstico y tratamiento, así como sus capacidades para la actualización médica. ${ }^{1}$

La Universidad Canadiense de McMaster, institución que adoptó esta nueva metodología en 1960, extiende la experiencia como foco central de la enseñanza de la medicina y establece un adecuado juicio de valor sobre la competencia y el desempeño del profesional de la medicina en la práctica clínica. Pero, el impulso fundamental lo da Cochrane A, quien reclama, en la década de los 70, una revisión crítica de todos los ensayos clínicos controlados, relevantes y periódicos, según especialidades. $^{2}$

Este movimiento resultó favorecido por el desarrollo de las nuevas tecnologías informáticas que contribuyen a modificar los modelos tradicionales de la atención médica, y desde el nacimiento, en 1989, de las denominadas "clinical practice guidelines" como respuesta a la necesidad de promover la eficiencia sanitaria; diversas acepciones como; guías, protocolos, vías clínicas, entre otros, se han extendido por servicios clínicos y hospitales promovidas por organizaciones médicas y sociedades profesionales.

Muchos han sido los grupo de autores en Cuba, que han escrito "Guías de Prácticas Clínicas", se recogen en la literatura más de 300 de diversas especialidades con la 


\section{Revista Cubana de Salud Pública. 2011;37(3):349-358}

utilización de métodos basados en opinión de expertos o basadas en el consenso, pero pocas de ellas pueden insertarse en el ámbito internacional al no estar basadas en las mejores evidencias científicas, como se hace en el resto del mundo.

Es una necesidad concentrar esfuerzos en elaborar guías de buena calidad en el contexto nacional para mejorar la atención médica.

Las guías de práctica clínica (GPC) tienen como objetivo fundamental el de reducir la variabilidad de la práctica médica para garantizar un nivel óptimo de calidad y mejorar la atención en salud, sus orígenes están basados en los fundamentos de la medicina basada en la evidencia y están dirigidas a profesionales y pacientes con el objetivo de proporcionar información para la toma de decisiones. Se sustentan en los estudios de revisiones sistemáticas de la literatura científica disponible y realizan recomendaciones para la actuación clínica según la evidencia que las respalda.

Internacionalmente es reconocido el trabajo realizado por varias organizaciones y redes científicas encaminadas a promover la elaboración, implementación y evaluación de las guías de práctica clínica y entre ellas se encuentra la Red I beroamericana de Guías de Práctica Clínica, que se conforma en el año 2004 en Porto Alegre, Brasil y tiene como objetivos:

1. Compartir información, instrumentos, herramientas y experiencias sobre la elaboración e implantación de guías de práctica clínica y calidad de los servicios sanitarios, entre los socios de 19 países.

2. Realizar cursos y talleres para socios, asistentes locales no socios y otras actividades de formación sobre calidad sanitaria y guías clínicas.

3. Diseñar y coordinar proyectos comunes de estudios e investigación para los tres próximos años.

4. Puesta en común de los avances alcanzados por los grupos de trabajos de la red.

5. Favorecer el intercambio de ciencia hecha en español y portugués.

6. Compartir y mejorar la comunicación a través de la plataforma virtual de la red, que es el instrumento de intercambio y la formación virtual.

Cuba forma parte de esta red desde su inicio.

La Red I beroamericana realiza la reunión anual en un país diferente cada vez de los que la integran y La Habana, Cuba fue su sede en el 2006.

La colaboración AGREE está constituida por un amplio grupo de instituciones académicas y agencias productoras de guías que ofrecen un instrumento de evaluación de calidad, como marco sistemático para el análisis de los componentes clave que determinan la calidad de las guías; incluye la elaboración y documentación del proceso, conocido como el Instrumento AGREE(Appaisal of guidelines research and evaluation), diseñado por investigadores de 13 países en función de crear una iniciativa internacional coordinada e identificar áreas potenciales de armonización para la elaboración de las guías. Consta de seis áreas teóricas de calidad con 23 criterios, y donde participan 195 evaluadores de 11 países y se trabaja con 100 guías en un primer momento y en un segundo momento de su creación participan 70 nuevos evaluadores y trabajan en una muestra aleatoria de tres guías, antes de obtener la versión final del instrumento. 
En Cuba existe un Programa Nacional de Guías de Practica Clínica desde el año 2005 coordinado por la Dirección Nacional de Ciencia y Técnica del Ministerio de Salud Pública.

El presente artículo tiene como objetivo evaluar la calidad de las GPC producidas en Cuba por las distintas especialidades utilizando para ello el instrumento AGREE, así como determinar las áreas de mayores dificultades en su elaboración.

\section{MÉTODOS}

Se realizó una búsqueda en los documentos impresos y en la Red Telemática de Salud de Cuba (Infomed) y con los grupos de especialidades, de todos los documentos identificados como Guías de Práctica Clínica o guías clínicas o guías prácticas de las diferentes especialidades médicas.

\section{Criterios de elegibilidad}

- Los documentos deben haber sido producidos por una o más instituciones o especialidades médicas existentes en Cuba.

- Los documentos deben haber sido producidos durante el período comprendido entre 1987 y 2007.

- Deben poder ser recuperados a texto completo.

\section{Criterios de inclusión}

Todos los documentos nombrados Guías de práctica clínica, que disponibles en el MINSAP, en la Red Telemática de Salud de Cuba o en las búsquedas en sitios web de instituciones de salud, esas últimas permiten identificar documentos difundidos exclusivamente en la web.

- De cualquiera de las especialidades médicas existentes en el país

\section{Criterios de exclusión}

- Documentos que NO contengan la referencia del año de elaboración.

- Documentos que cumplan la definición de protocolo de actuación.

Para cumplir con los objetivos de este estudio se conformó el grupo evaluador integrado por cuatro especialistas, los cuales fueron capacitados en el conocimiento y aprendizaje del instrumento AGREE para que pudieran realizar la evaluación de GPC mediante su utilización.

De cada guía se recogió el criterio de tres evaluadores y en el caso de resultados discordantes fue evaluada por otros dos evaluadores independientes.

Las seis áreas o aspectos considerados en la evaluación (anexo) fueron:

1. Alcance y objetivos. 


\section{Revista Cubana de Salud Pública. 2011;37(3):349-358}

2. Participación de los implicados.

3. El rigor en la elaboración.

4. La claridad y presentación.

5. Aplicabilidad.

6. La independencia editorial, teniendo al final una valoración global sobre lo que aporta.

En el aspecto Evaluación global (anexo), se especifican las recomendaciones de la guía evaluada para su uso en la práctica.

Estas son las seis áreas de calidad, cada una con varios ítems, donde el mayor número está relacionado con el rigor en la elaboración. ${ }^{3}$

Existe un grupo de atributos deseables a revisar en toda GPC y su confluencia en una guía le daría mucha credibilidad, tanto a sus recomendaciones como a su potencial utilización, ${ }^{4}$ lo que supondría la obtención de los resultados clínicos deseados, ellos son:

- Elaboración multidisciplinaria: deben participar todos los miembros que puedan estar implicados en la práctica clínica del tema objeto de la guía (médicos de atención primaria y especializada, enfermería, otros trabajadores sanitarios o no sanitarios, pacientes, organizaciones de salud).

- Claridad: utilizar un lenguaje claro, definir los términos de modo preciso con una presentación lógica y fácil de seguir.

- Reproducibilidad: si se realizan todos los pasos indicados en la elaboración de la guía y la misma búsqueda bibliográfica con la circunstancia clínica referida, los resultados de las recomendaciones serían las mismas y la aplicación similar.

- Flexibilidad: deben reflejarse las alternativas de manejos razonables y posibles.

- Aplicabilidad: las recomendaciones propuestas pueden llevarse a la práctica en cualquier lugar o circunstancias o adecuarlas.

- Válidez: las recomendaciones propuestas son fruto de una revisión bibliográfica rigurosa.

- Actualización: aporta sistemáticamente las últimas novedades sobre ese tema.

\section{RESULTADOS}

Se revisaron 377 documentos, de ellos:

- Existe un importante grupo que no son GPC y aunque se publican con ese nombre son protocolos de actuación de los servicios elaborados por grupos de expertos o por consenso, donde no se utilizaron las mejores evidencias, 4, 5 sino las mejores

http://scielo.sld.cu 
experiencias. Tal el caso de un grupo de GPC que aparecen en las web de hospitales con el nombre de Guías, que no han sido aprobadas por la Dirección Nacional de Ciencia y Técnica, ni por el Buró Regulatorio para la Protección a la Salud.

- Se localizaron también otros documentos que aparecen impresos o en la red telemática, como "Guías de Buenas Prácticas Clínicas", nombre que se ha sido acordado al conjunto de obligaciones de los promotores, monitores e investigadores que participan en un ensayo clínico para una serie de procedimientos o normas diseñados con el fin de evitar errores y fraudes, así como garantizar que los sujetos de una investigación clínica mantengan íntegros sus derechos durante la misma,

- Los documento que tienen nombre de GPC y que fueron evaluados, tienen en común que no utilizaron la evidencia científica ${ }^{4,5}$ para su elaboración, fueron tan solo elaboradas por los grupos de expertos.

- Otro grupo de documentos con alta participación de la Sociedad Cubana de Cirugía Pediátrica, son documentos normativos o actualizaciones terapéuticas del tema pero se han publicado como GPC.

Otras debilidades encontradas y que aparecen reflejadas en la mayor parte de los documentos evaluados fueron:

- No están realizadas por equipos multidisciplinarios, sino sólo por un grupo de especialistas afines.

- No se tuvo en cuenta el punto de vista de los pacientes.

- No cuentan con información para pacientes, solo para uso de los profesionales.

- No tienen declarada fecha de actualización o revisión.

- Las recomendaciones no están refrendadas por las mejores evidencias ni existe claridad en las mismas.

- No dan respuesta a preguntas clínicas.

- No declaran los objetivos de la guía, ni la población diana, no tienen criterios de inclusión y exclusión.

- No se tuvo en cuenta el punto de vista de los pacientes.

Hay un grupo de documentos elaborados por expertos, que una vez evaluados no se corresponden con GPC y no son recomendados como tal y existe la metodología de elaboración para las GPC de enfermedad cerebrovascular, las de tratamiento inmunomodulador e inmunosupresor para la esclerosis múltiple, las de fractura de cadera en el adulto mayor, enfermedad de Parkinson y las guías de pesquisaje, diagnóstico y tratamiento de la diabetes mellitus tipo $2 .^{6}$

En estos momentos se trabaja en la construcción de las Directrices Metodológicas para la Elaboración y Adaptación de Guías de Práctica Clínica en el Sistema Nacional de Salud, para el trabajo de los grupos de autores y existen varios grupos multidisciplinarios que trabajan en la elaboración o adaptación de guías, entre las cuales se encuentra la GPC de profilaxis de la trombosis venosa profunda. 
Revista Cubana de Salud Pública. 2011;37(3):349-358

\section{Anexo}

Evaluación de la calidad de las guías de práctica clínica cubanas

$\begin{array}{lccc}\text { Título } & \text { de } & \text { la } & \text { guía: } \\ \text { Año } & \text { de } & & \text { realización: } \\ \text { Centro } & \text { que } & \text { la } & \text { elabora: }\end{array}$

Coordinador:

\begin{tabular}{|c|c|c|c|c|c|c|c|}
\hline \multicolumn{8}{|c|}{ Alcance y objetivos } \\
\hline 1 & $\begin{array}{l}\text { El (los) objetivos(s) general (es) de la Guía } \\
\text { está( } n \text { ) específicamente descritos. }\end{array}$ & $\begin{array}{l}\text { Muy de } \\
\text { acuerdo }\end{array}$ & 4 & 3 & 2 & 1 & $\begin{array}{l}\text { Muy en } \\
\text { desacuerdo }\end{array}$ \\
\hline 2 & $\begin{array}{l}\text { El (los) aspecto (s) clínico(s) por la Guía } \\
\text { está( } n \text { ) específicamente descritos. }\end{array}$ & $\begin{array}{l}\text { Muy de } \\
\text { acuerdo }\end{array}$ & 4 & 3 & 2 & 1 & $\begin{array}{l}\text { Muy en } \\
\text { desacuerdo }\end{array}$ \\
\hline 3 & $\begin{array}{l}\text { Los pacientes a quienes se pretende aplicar la } \\
\text { Guía están específicamente descritos. }\end{array}$ & $\begin{array}{l}\text { Muy de } \\
\text { acuerdo }\end{array}$ & 4 & 3 & 2 & 1 & $\begin{array}{l}\text { Muy en } \\
\text { desacuerdo }\end{array}$ \\
\hline \multicolumn{8}{|c|}{ Participación de los implicados } \\
\hline 4 & $\begin{array}{l}\text { El grupo que desarrolla la Guía incluye } \\
\text { individuos de todos los grupos profesionales } \\
\text { relevantes. }\end{array}$ & $\begin{array}{l}\text { Muy de } \\
\text { acuerdo }\end{array}$ & 4 & 3 & 2 & 1 & $\begin{array}{l}\text { Muy en } \\
\text { desacuerdo }\end{array}$ \\
\hline 5 & $\begin{array}{l}\text { Se han tenido en cuenta los puntos de vista del } \\
\text { paciente y sus preferencias. }\end{array}$ & $\begin{array}{l}\text { Muy de } \\
\text { acuerdo }\end{array}$ & 4 & 3 & 2 & 1 & $\begin{array}{l}\text { Muy en } \\
\text { desacuerdo }\end{array}$ \\
\hline 6 & $\begin{array}{l}\text { Los usuarios diana de la Guía están claramente } \\
\text { definidos. }\end{array}$ & $\begin{array}{l}\text { Muy de } \\
\text { acuerdo }\end{array}$ & 4 & 3 & 2 & 1 & $\begin{array}{l}\text { Muy en } \\
\text { desacuerdo }\end{array}$ \\
\hline 7 & $\begin{array}{l}\text { La Guía ha sido probada entre los usuarios } \\
\text { diana. }\end{array}$ & $\begin{array}{l}\text { Muy de } \\
\text { acuerdo }\end{array}$ & 4 & 3 & 2 & 1 & $\begin{array}{l}\text { Muy en } \\
\text { desacuerdo }\end{array}$ \\
\hline \multicolumn{8}{|c|}{ Rigor en la elaboración } \\
\hline 8 & $\begin{array}{l}\text { Se han utilizado métodos sistemáticos para la } \\
\text { búsqueda de evidencia. }\end{array}$ & $\begin{array}{l}\text { Muy de } \\
\text { acuerdo }\end{array}$ & 4 & 3 & 2 & 1 & $\begin{array}{l}\text { Muy en } \\
\text { desacuerdo }\end{array}$ \\
\hline 9 & $\begin{array}{l}\text { Los criterios para seleccionar la evidencia se } \\
\text { describen con claridad. }\end{array}$ & $\begin{array}{l}\text { Muy de } \\
\text { acuerdo }\end{array}$ & 4 & 3 & 2 & 1 & $\begin{array}{l}\text { Muy en } \\
\text { desacuerdo }\end{array}$ \\
\hline 10 & $\begin{array}{l}\text { Los métodos utilizados para formular las } \\
\text { recomendaciones están claramente definidos. }\end{array}$ & Muy de & 4 & 3 & 2 & 1 & Muy en \\
\hline
\end{tabular}


Revista Cubana de Salud Pública. 2011;37(3):349-358

\begin{tabular}{|c|c|c|c|c|c|c|c|}
\hline & & acuerdo & & & & & desacuerdo \\
\hline 11 & $\begin{array}{l}\text { Al formular las recomendaciones han sido } \\
\text { considerados los beneficios en salud, los } \\
\text { efectos secundarios y los riesgos. }\end{array}$ & $\begin{array}{l}\text { Muy de } \\
\text { acuerdo }\end{array}$ & 4 & 3 & 2 & 1 & $\begin{array}{l}\text { Muy en } \\
\text { desacuerdo }\end{array}$ \\
\hline 12 & $\begin{array}{l}\text { Hay relación explicita entre cada una de las } \\
\text { recomendaciones y las evidencias en las que se } \\
\text { basan. }\end{array}$ & $\begin{array}{l}\text { Muy de } \\
\text { acuerdo }\end{array}$ & 4 & 3 & 2 & 1 & $\begin{array}{l}\text { Muy en } \\
\text { desacuerdo }\end{array}$ \\
\hline 13 & $\begin{array}{l}\text { La Guía ha sido revisada por expertos externos } \\
\text { antes de su publicación. }\end{array}$ & $\begin{array}{l}\text { Muy de } \\
\text { acuerdo }\end{array}$ & 4 & 3 & 2 & 1 & $\begin{array}{l}\text { Muy en } \\
\text { desacuerdo }\end{array}$ \\
\hline 14 & $\begin{array}{l}\text { Se incluye un procedimiento para actualizar la } \\
\text { Guía. }\end{array}$ & $\begin{array}{l}\text { Muy de } \\
\text { acuerdo }\end{array}$ & 4 & 3 & 2 & 1 & $\begin{array}{l}\text { Muy en } \\
\text { desacuerdo }\end{array}$ \\
\hline \multicolumn{8}{|c|}{ Claridad y presentación } \\
\hline 15 & $\begin{array}{l}\text { Las recomendaciones son específicas y no son } \\
\text { ambiguas. }\end{array}$ & $\begin{array}{l}\text { Muy de } \\
\text { acuerdo }\end{array}$ & 4 & 3 & 2 & 1 & $\begin{array}{l}\text { Muy en } \\
\text { desacuerdo }\end{array}$ \\
\hline 16 & $\begin{array}{l}\text { Las distintas opciones para el manejo de la } \\
\text { enfermedad o condición se presentan } \\
\text { claramente. }\end{array}$ & $\begin{array}{l}\text { Muy de } \\
\text { acuerdo }\end{array}$ & 4 & 3 & 2 & 1 & $\begin{array}{l}\text { Muy en } \\
\text { desacuerdo }\end{array}$ \\
\hline 17 & $\begin{array}{l}\text { Las recomendaciones claves son fácilmente } \\
\text { identificables. }\end{array}$ & $\begin{array}{l}\text { Muy de } \\
\text { acuerdo }\end{array}$ & 4 & 3 & 2 & 1 & $\begin{array}{l}\text { Muy en } \\
\text { desacuerdo }\end{array}$ \\
\hline 18 & $\begin{array}{l}\text { La Guía se apoya con herramientas para su } \\
\text { aplicación. }\end{array}$ & $\begin{array}{l}\text { Muy de } \\
\text { acuerdo }\end{array}$ & 4 & 3 & 2 & 1 & $\begin{array}{l}\text { Muy en } \\
\text { desacuerdo }\end{array}$ \\
\hline \multicolumn{8}{|c|}{ Aplicabilidad } \\
\hline 19 & $\begin{array}{l}\text { Se han discutido las barreras organizativas } \\
\text { potenciales a la hora de aplicar las } \\
\text { recomendaciones. }\end{array}$ & $\begin{array}{l}\text { Muy de } \\
\text { acuerdo }\end{array}$ & 4 & 3 & 2 & 1 & $\begin{array}{l}\text { Muy en } \\
\text { desacuerdo }\end{array}$ \\
\hline 20 & $\begin{array}{l}\text { Han sido considerados los costes potenciales de } \\
\text { la aplicación de las recomendaciones. }\end{array}$ & $\begin{array}{l}\text { Muy de } \\
\text { acuerdo }\end{array}$ & 4 & 3 & 2 & 1 & $\begin{array}{l}\text { Muy en } \\
\text { desacuerdo }\end{array}$ \\
\hline 21 & $\begin{array}{l}\text { La guía ofrece una relación de criterios clave } \\
\text { con el fin de realizar monitorización y/o } \\
\text { auditoria. }\end{array}$ & $\begin{array}{l}\text { Muy de } \\
\text { acuerdo }\end{array}$ & 4 & 3 & 2 & 1 & $\begin{array}{l}\text { Muy en } \\
\text { desacuerdo }\end{array}$ \\
\hline \multicolumn{8}{|c|}{ Independencia editorial } \\
\hline 22 & La Guía es editorialmente independiente de la & Muy de & 4 & 3 & 2 & 1 & Muy en \\
\hline
\end{tabular}


Revista Cubana de Salud Pública. 2011;37(3):349-358

\begin{tabular}{|l|l|l|l|l|l|l|l|}
\hline & entidad financiadora. & acuerdo & & & & & desacuerdo \\
\hline 23 & $\begin{array}{l}\text { Se han registrado los conflictos de intereses de } \\
\text { los miembros del grupo de desarrollo. }\end{array}$ & Muy de & & & & & Muy en \\
\hline Evaluación global & 4 & 3 & 2 & 1 & \\
\hline \multicolumn{2}{|l|}{ ¿Recomendaría está Guía para su uso en la Práctica? } & & & \\
\hline Muy Recomendada. & & & \\
\hline Recomendada (con condiciones o modificaciones). & & & \\
\hline Recomendada. & &
\end{tabular}

\section{REFERENCI AS BI BLI OGRÁFICAS}

1. Hernández Rodríguez A. Las Guías de Práctica Clínica en la Atención Médica. Rev Cubana Ortop Traumatol [Internet]. 2008 [citado 18 Dic 2008].;22(2). Disponible en: http://scielo.sld.cu/scielo.php?script=sci_arttext\&pid=S0864215X2008000200006\&lng=es

2. Lede R, Abriata G, Copertari P. La medicina basada en evidencias: un movimiento cultural a favor de una mejor asistencia médica. [Internet]. [citado 18 Dic 2008]. Disponible en: http://scielo. sld.cu/scieloOrg/php/reference. $\mathrm{php}$ ?pid=S1024$94352003000300002 \&$ caller $=$ scielo. sld.cu\&lang=en

3. The AGREE Collaboration. AGREE Instrument Spanish versión [Internet]. [citado 27 Ene 2006]. Disponible en: http://www.agreecollaboration.org/

4. Frezno Cháves C. Medicina basada en evidencias. Rev Cubana Med Gen Integr [Internet]. 2001 [citada 9 Ene 2006];17(2). Disponible en:

http://scielo.sld.cu/scielo.php?script=sci_arttext\&pid=S0864$\underline{21252001000200014 \& \operatorname{lng}=e s \& n r m=i s o \& \operatorname{lng}=e s}$

5. Boucourt Rivera L. Su excelencia: la medicina basada en evidencias. ACIMED. 2003; 11(3): 3-4.

6. Aymerich M. Del conocimiento científico de la investigación clínica a la cabecera del enfermo: las guías de práctica clínica y su implementación. Gac Sanit. 2004; 18(4): 326-34.

Recibido: 13 de octubre de 2010.

Aprobado: 24 de enero de 2011. 
Ana Margarita Toledo Fernández. Metodóloga de la Dirección Nacional de Ciencia y Técnica. Calle I, No 202 esquina a Línea, Edificio B del MINSAP. La Habana, Cuba. Correo electrónico: amtoledo@infomed.sld.cu 